\title{
Efficient Variable Thickness Radome Design with Insertion Phase Delay Correction
}

\author{
Wanye Xu $\mathbb{D}^{1,2}$ Peng Li, ${ }^{1,2}$ and Yuanying Qiu ${ }^{3}{ }^{3}$ \\ ${ }^{1}$ Key Laboratory of Electronic Equipment Structure Design, Ministry of Education, Xidian University, Xi'an, \\ Shaanxi 710071, China \\ ${ }^{2}$ Shaanxi Key Laboratory of Space Solar Power Station System, Xidian University, Xi'an, Shaanxi 710071, China \\ ${ }^{3}$ National Key Laboratory of Antenna and Microwave Technology, Xidian University, Xi'an, Shaanxi 710071, China \\ Correspondence should be addressed to Wanye Xu; xuwanye@163.com
}

Received 24 September 2019; Accepted 1 November 2019; Published 16 November 2019

Academic Editor: Luca Catarinucci

Copyright (C) 2019 Wanye Xu et al. This is an open access article distributed under the Creative Commons Attribution License, which permits unrestricted use, distribution, and reproduction in any medium, provided the original work is properly cited.

\begin{abstract}
Variable thickness design is capable of yielding excellent electromagnetic (EM) performance for streamlined airborne radomes. The traditional optimization method using evolutionary computation (EC) techniques can yield variable thickness radome (VTR) design with optimal EM performance but uncontrollable thickness profile, leading to manufacturing difficulties. Thickness profile design based on average incident angle is an efficient way for VTR design with smooth thickness profile, whereas the boresight error (BSE) is relatively large. In this work, insertion phase delay (IPD) correction is introduced in the efficient thickness profile design process to realize a better balance between EM performance and thickness profile smoothness of airborne radomes. The thickness profile obtained from average incident angle is adjusted for IPD distribution symmetry with respect to the antenna axis under scan angles with large BSE. Results indicate that the proposed method can effectively improve the BSE characteristics at the expense of slight deterioration of computational efficiency and thickness profile smoothness.
\end{abstract}

\section{Introduction}

Radomes are used in various applications to protect antennas such as reflector antennas and phased array antennas. [1-4]. Variable thickness design is effective in improving the electromagnetic (EM) performance of airborne radomes which is prone to be deteriorated by the adverse streamlined shapes [5-11]. Through thickness profile optimization using evolutionary computation (EC) techniques, such as particle swarm optimization (PSO) and genetic algorithm (GA), variable thickness radomes (VTRs) can yield excellent EM performance, such as transmission loss (TL) and boresight error (BSE) [7-11]. The main drawbacks of the optimization method are the concomitant large computational cost in searching for the optimal solution and the radically varying thickness profile which is difficult for manufacturing.

Incident angle is an important factor in determining the transmission characteristics of radomes. Streamlined radome shapes introduce asymmetric incident angle distribution varying during antenna scanning, which tends to degrade the EM performance. Through analyzing the incident angle variation characteristics for different radome areas, thickness can be accordingly designed to compensate the adverse effect of incident angle. Average incident angle with proper modification is effective in efficiently designing the thickness profile through the half-wave thickness expression [12]. Smooth thickness profile is guaranteed by the continuous radome shape which results in the gradual variation of average incident angle. This method can yield satisfactory TL, whereas the BSE, although good and acceptable, is still about 3 times the optimal BSE obtained by the optimization method [12].

Asymmetric insertion phase delay (IPD) is the main cause of radome BSE. Essentially, the half-wave thickness implies optimal transmission efficiency and gives little consideration to BSE characteristics. In this work, on the 
basis of the thickness profile obtained by the average incident angle method in [12], radome IPD under scan angles with large BSE is corrected for symmetry w.r.t. the antenna axis, thus improving the BSE characteristics. The tangent-ogival radome in [12] is used to validate the effectiveness of the proposed method.

\section{Insertion Phase Delay (IPD) Correction}

Through changing the radome thickness, the IPD correction is meant to improve the IPD distribution under particular antenna scan angles, thus reducing the BSE. As IPD asymmetry is the main cause of radome BSE, it is considered to realize IPD distribution symmetric to antenna aperture center. The IPD caused by the radome longitudinal curvature along the thickness profile is the main target of symmetry, whereas the effect of radome lateral curvature on IPD is further considered through scale factor. At different antenna scan angles, the required radome thickness profiles can be different. The IPD correction is therefore a compromise of optimal thickness value under different scan angles to yield minimum BSE.

As in [12], the $3 \mathrm{D}$ ray-tracing method is employed to evaluate the EM performance parameters of the antennaradome system. This method assumes that the EM fields radiate from the antenna within the cylindrical area confined by the aperture and transmit through the radome as the wall is locally plane at each intercept point. Radome effects involve amplitude attenuation and phase distortion of the aperture field.

Radome IPD, cause of the phase distortion, depends on 3 parameters of radome: thickness, permittivity, and shape (which relates to the incident angle and polarization angle). As shown in Figure 1(a), at different scan angles, the antenna sees different radome parts with different radome shape and thus incident/polarization angle distribution varies during antenna scanning. At a particular scan angle, asymmetric radome shape will introduce asymmetric IPD distribution, as shown in Figure 1(b). Through analyzing the average incident angle of radome points on the shape profile, thickness profile with high transmission efficiency can be obtained [12]. As the IPD distribution is not involved in this method, radome BSE can be large, and a modification factor for the average incident angle has been introduced to reduce the BSE [12]. Even so, the BSE is still much larger than the optimal value of the optimization method.

In fact, for a particular scan angle, the corresponding IPD distribution can be changed through thickness adjustment to reduce the BSE. Figure 2(a) shows the IPD curve as red full line and its mirror symmetry curve as blue dashed line. With the horizontal black dashed line through the center of both curves as the boundary, we can obtain in the upper area the symmetric curve I and in the lower area the symmetric curve II. The IPD curve can be corrected to be curve I (right side correction, RSC) or curve II (left side correction, LSC). With RSC as example, Figure 2(b) shows the correction with scale factor, which is meant to further improve the IPD distribution.
Figure 3 plots the 3D case of antenna-radome system. The radome generatrix in Figure 1 (a) can be rotated around the radome axis to form the $3 \mathrm{D}$ model. For a specified point on the radome generatrix, as lateral curvature effect exists, the two points on the longitudinal shape profile symmetric with the middle point can have different incident angle characteristics. Therefore, if the two symmetric points are set to have the same IPD, as the lateral IPD distribution can still be different, the overall IPD distribution may not be the best. To minimize the IPD difference within the whole aperture, a scale factor can be applied. As shown in Figure 2(b), from the middle point to the left/right boundary point, the scale factor varies linearly form 1 to a specified value.

Radome IPD can be calculated via the following formula:

$$
\mathrm{IPD}=\frac{2 \pi}{\lambda} d\left(\sqrt{\varepsilon_{r}-\sin ^{2} \theta}-\cos \theta\right)
$$

where $\lambda$ is the wavelength, $d$ is the radome wall thickness, $\varepsilon_{r}$ is the relative permittivity of radome material, and $\theta$ is the incident angle.

Radome IPD can be modified to be a particular value through thickness adjustment. As shown in Figure 2, if IPD $_{1}$ needs to be modified to be IPD 2 , the thickness after adjustment can be computed by

$$
\begin{gathered}
d_{2}=\frac{\mathrm{IPD}_{2}}{\mathrm{IPD}_{1}} d_{1}, \\
\text { or } d_{2}=\operatorname{IPD}_{2} \frac{\lambda}{2 \pi\left(\sqrt{\varepsilon_{r}-\sin ^{2} \theta}-\cos \theta\right)} .
\end{gathered}
$$

The thickness profile can then be obtained by cubic-Bspline interpolation [7].

From a given thickness profile, the BSE w.r.t. antenna scan angle can be calculated. Then, the 2D case IPD curve under the scan angle corresponding to the maximum BSE can be changed. It should be noticed that the changed thickness profile will affect the EM performance under some other scan angles. As shown in Figure 4, for the antennaradome system shown in Figure 1(a), there exists a scan angle range affected by the changed thickness profile within the area illuminated by the antenna aperture. For LSC, the affected scan angle range (ASAR) is $\left[\theta_{\min }^{L}, \theta_{\text {max }}^{L}\right]$, and for RSC, $\left[\theta_{\min }^{R}, \theta_{\max }^{R}\right]$. Figure 4 shows the LSC case, and the RSC case has similar results. Therefore, in determining the optimal scale factor, it would be a better choice to select both the side (left or right) and the scale factor which leads to the thickness profile with the minimum maximum BSE within ASAR.

For a set of scale factors $S_{i}, i=1,2, \ldots, p$, this can be formulated as

$$
\min _{L / R}\left[\begin{array}{c}
\min _{S} \max _{\theta^{L}} \operatorname{BSE}\left(\theta_{\min }^{L}, \ldots, \theta_{\max }^{L}, S_{1}, S_{2}, \ldots, S_{p}\right), \\
\min _{S} \max _{\theta^{R}} \operatorname{BSE}\left(\theta_{\min }^{R}, \ldots, \theta_{\max }^{R}, S_{1}, S_{2}, \ldots, S_{p}\right)
\end{array}\right] .
$$

The inner "max" operator is to find the maximum BSE for all scan angles within $\left[\theta_{\min }^{L}, \theta_{\max }^{L}\right]$, or $\left[\theta_{\min }^{R}, \theta_{\max }^{R}\right]$, with a 


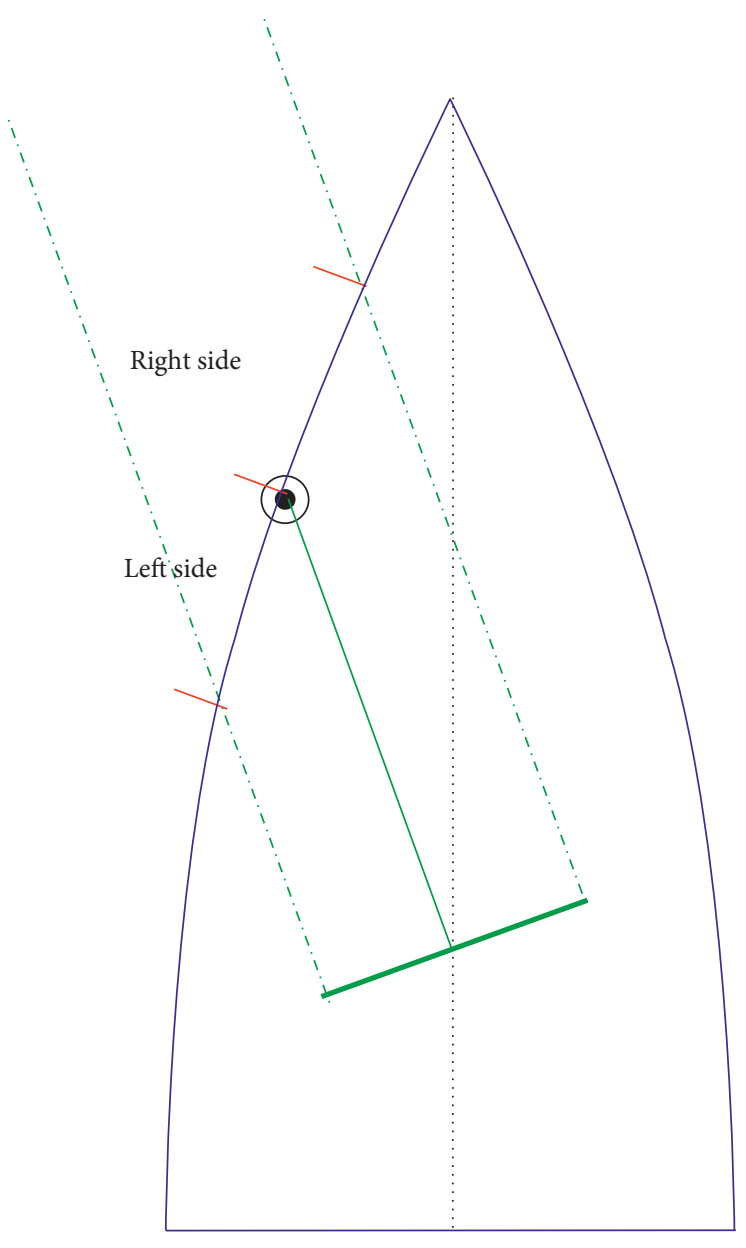

(a)

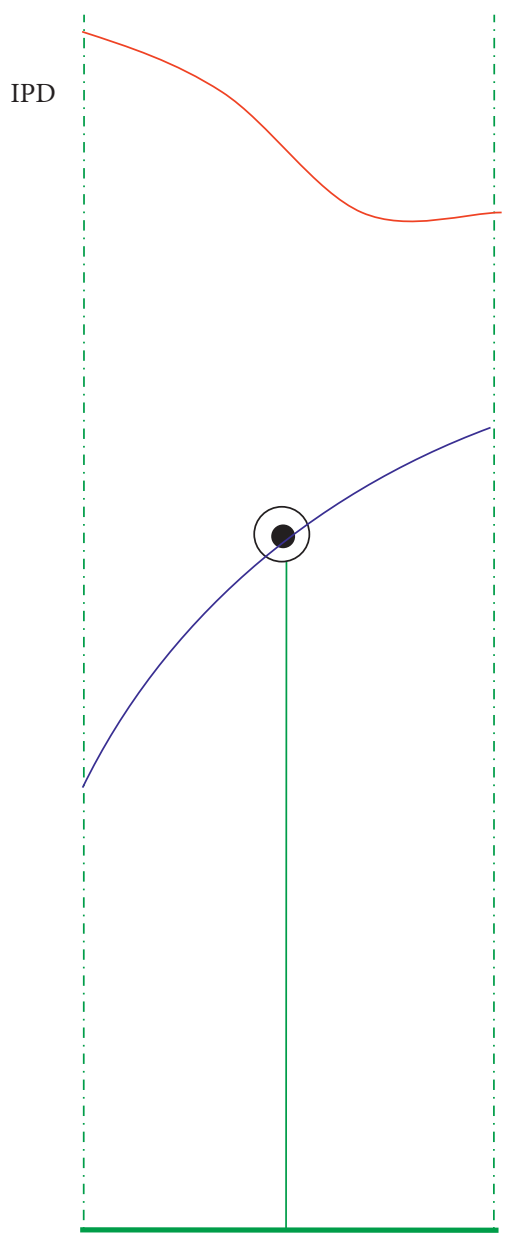

(b)

FIgURE 1: 2D case of the antenna-radome system. (a) Overall view. (b) Partial view with IPD.

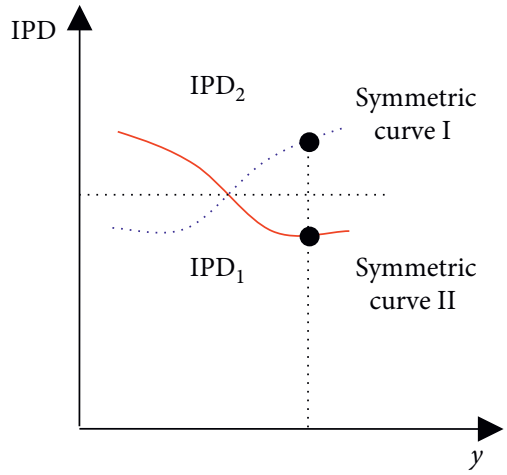

(a)

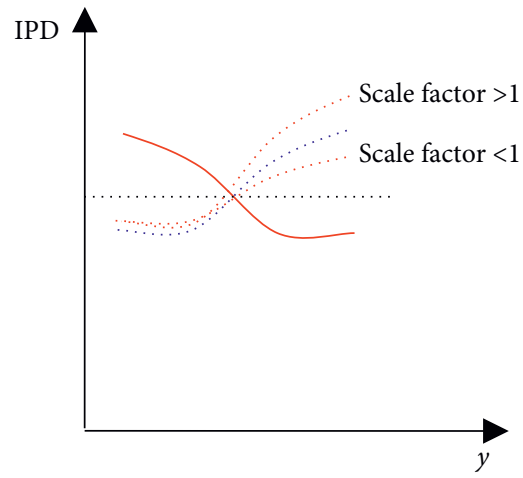

(b)

FIGURE 2: IPD correction for symmetry. (a) Without scale factor. (b) With scale factor for right side correction.

fixed scale factor, and the inner "min" operator yields the minimum value of the maximum BSE for all scale factors, and finally, the outer "min" operator gives the optimal BSE from the left- and right-side results. The output is the scale factor and the side (left or right) relevant to the minimum maximum BSE within ASAR.
The IPD correction procedure can be summarized as follows:

(1) Calculate the BSE curve of a given thickness profile (using the average incident angle method in [12], for example); 


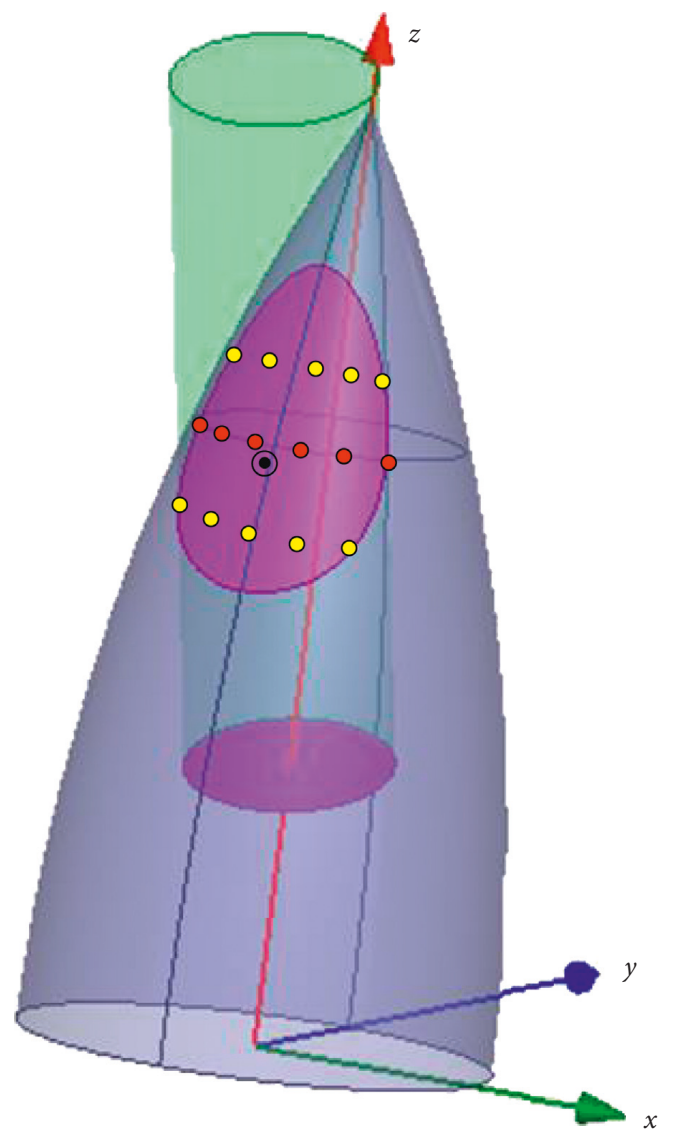

Figure 3: 3D case of antenna-radome system.

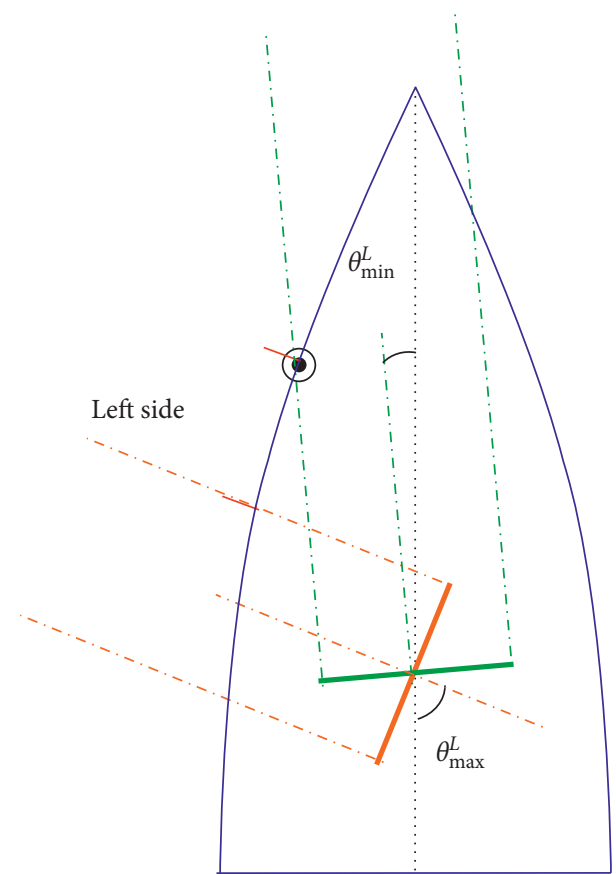

FIgURE 4: The scan angle range affected by a part of the thickness profile.

(2) Find the optimal scale factor and side (left or right) within ASAR using (4), and obtain a new thickness profile using (2) or (3);
(3) Return to step 1 with the new thickness profile and repeat the IPD correction. When the maximum BSE after IPD correction is no less than the original value, the procedure can be terminated.

It should be noticed that even if at one iteration, the maximum BSE is not reduced by the IPD correction and further correction may yield smaller maximum BSE. Thus, it is suggested to calculate the correction iteration by 5 times and then start the termination judgment. In this way, the thickness design procedure can be refined as the flow chart shown in Figure 5.

\section{Results}

The tangent-ogival radome used in [12] is employed to validate the proposed design method. As shown in Figure 6, the circular-aperture antenna is working at $9.4 \mathrm{GHz}$ and has a linearly polarized aperture field. The radome is made of glass composite with permittivity and loss tangent as 4 and 0.015 , respectively, and coated with a typical paint with permittivity and loss tangent as 3.46 and 0.068 , respectively. The thickness of the paint is $0.2 \mathrm{~mm}$ whereas the thickness of the glass composite needs to be designed.

Verification of the EM performance analysis method can be found in [12], where the computed results are compared with the computed and measured results in [5] and [13]. In [12], the thickness is designed based on the average incident angle with a modification factor (which is for the BSE). Results show that the method in [12] can yield smooth thickness profile with similar TL and inferior BSE, compared with the optimization method. In the following, the final results of the proposed efficient design will be given and compared with the previous results. The iteration process in determining the optimal scale factor and side is shown to help understand the effect of the proposed method. As the modification factor in [12] and the proposed side selection both affect the computational cost (number of EM performance evaluation), LSC and RSC are further discussed to show the importance of the side selection strategy, and finally, the necessity of the modification factor is shown.

3.1. Efficient Design with IPD Correction. The scale factor range is set as $[0.9,1.1]$ with a step of 0.01 . In IPD correction, it is necessary to properly discretize the thickness profile into a series of points along radome height. If the discrete points are too sparse, the precision of IPD symmetry in LSC or RSC will degrade, whereas the final thickness profile is prone to be ragged when the points are too dense. For the case in this work, a step of $0.04 \mathrm{~m}$ or $0.03 \mathrm{~m}$ will be a good compromise between IPD correction precision and thickness profile smoothness. To realize the best results, the $0.04 \mathrm{~m}$ step is mainly employed, i.e., $1,0.96,0.92, \ldots, 0.28,0.24$, and 0.19 (unit: $\mathrm{m}$ ), whereas in Section 3.2, the $0.03 \mathrm{~m}$ step is employed for the no-modification-factor case, i.e., $1,0.97,0.94, \ldots$, $0.22,0.19$ (unit: $\mathrm{m}$ ).

Based on the 3D design method with an incident angle modification factor of 0.75 in [12], the thickness profile is further adjusted using the proposed IPD correction method. 


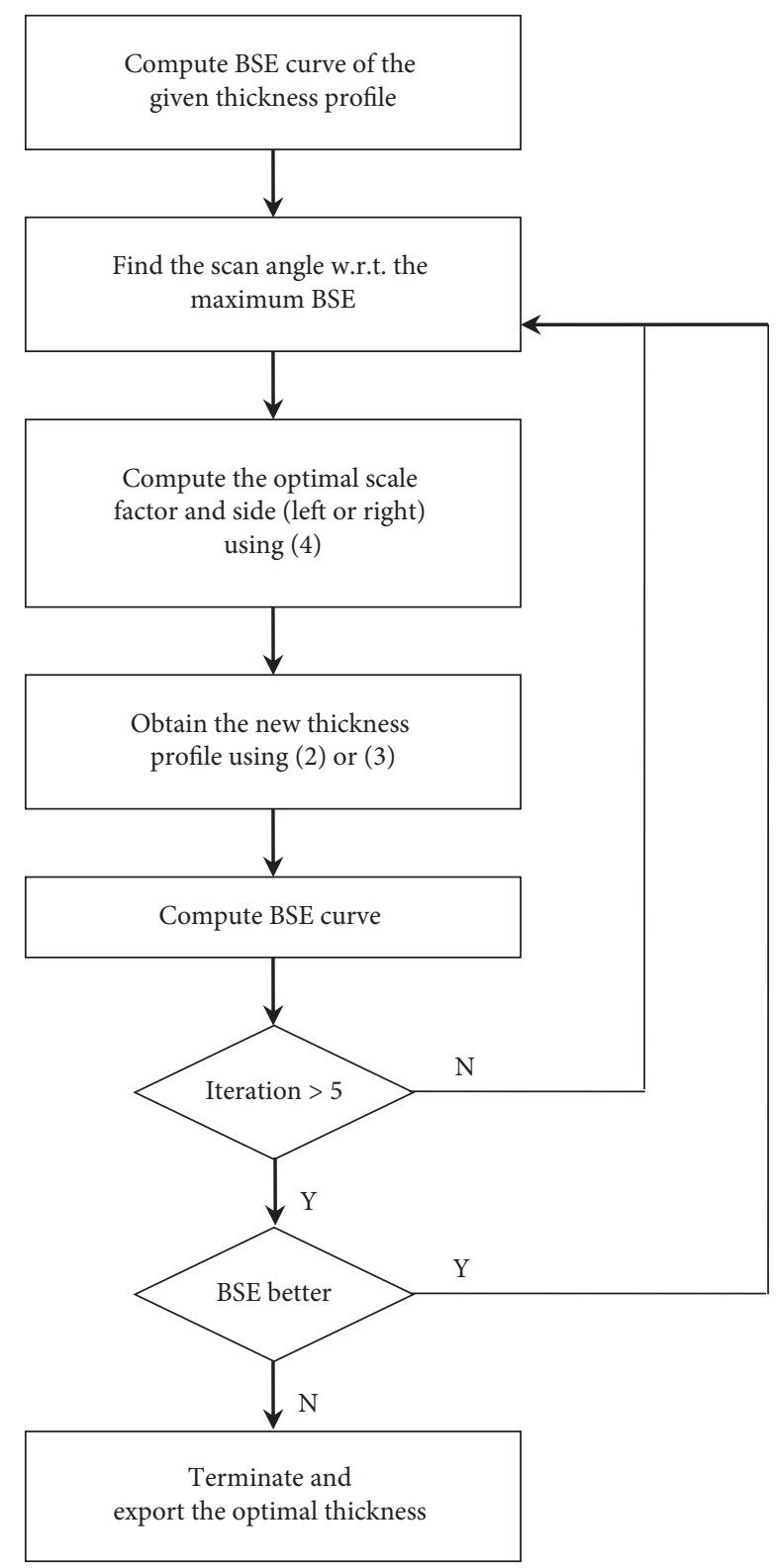

Figure 5: Efficient thickness design procedure.

The IPD correction is iterated for 7 times, and the corresponding maximum BSE is listed in Table 1. In each iteration, each scale factor within [0.9, 1.1] (step: 0.01) is imposed on both LSC and RSC, and the corresponding EM performance is then computed, resulting in $21 \times 2 \times 7=294$ calculations of the EM performance, which is the main source of the computational cost (more than 99\% of the design process). One calculation of the EM performance takes about 10 seconds using a computer with $3.1 \mathrm{GHz}$ processor (Intel i3). Nevertheless, as only the ASAR needs to be considered, the calculation is not a "full" EM performance evaluation which involves all scan angles within $\left[0^{\circ}, 90^{\circ}\right]$ (step: $2^{\circ}$ ). The mean ASAR considering every scan angle and both LSC and RSC is only about $60 \%$ of the whole scan angles. The equivalent "full" EM performance evaluation in the IPD correction process is about 176. Along with the 61
EM performance evaluation in determining the average incident angle modification factor [12], the overall times of EM performance evaluation is considered as 237 .

As shown in Table 1, the optimal BSE arises at the 6th iteration with maximum BSE as $0.706 \mathrm{mrad}$. Figure 7 plots the thickness profile of the optimization method, the previous efficient design in [12], and the efficient design proposed in this work. Figure 8 shows the EM performance comparison of different methods, and Table 2 gives the corresponding EM performance and the computational cost.

It can be seen from these results that the proposed efficient design method, compared with the previous one, can yield much better BSE (maximum value improved by about $50 \%$ ) with slightly degraded thickness profile (smooth, but not as smooth as the previous one). The maximum BSE of the proposed method is close to that of the optimization method. All three methods yield similar TL characteristics, which is an indication of the insensitivity of TL to slight thickness variation. With the optimization method as reference, the efficiency of the efficient design is only deteriorated from $0.6 \%$ for the previous one to $2.4 \%$ for the present one. The proposed efficient design method can yield much better BSE with slightly degraded thickness profile smoothness and computational efficiency.

To show the effect of the IPD correction method, the thickness profile and the corresponding BSE characteristics in the iteration process are shown in Figure 9 in a step-bystep way. The TL nearly stays the same in the process and is therefore omitted. It can be observed that, at each iteration, a part of the thickness profile is adjusted from IPD correction, which affects a part of the BSE curve. Maximum BSE is reduced continuously, and the final thickness profile and BSE curve are the result of all these steps.

3.2. Effect of Side Selection and Modification Factor. In the design process, the selection of which side to be modified is implemented by computing both sides and choosing the one with the optimal BSE characteristics in each iteration. This is an important part of the computational cost. It would be interesting to see what happens when the side (either left or right) is fixed in the process.

Table 3 lists the maximum BSE during iteration when only one side correction is considered. The iteration terminates at the 8th iteration for both cases, and the optimal BSE is $1.363 \mathrm{mrad}$ for LSC and $0.918 \mathrm{mrad}$ for RSC, respectively. RSC can yield better results than LSC. Compared with the results in the previous subsection, RSC only yields larger maximum BSE (from $0.706 \mathrm{mrad}$ to $0.918 \mathrm{mrad}$ ) with the EM performance computation reduction from 237 to 149 times. Altogether, it is considered that the BSE characteristics are more important than the computational cost here, and the LSC-RSC method is considered as better than one-side-only.

Besides, the modification factor for average incident angle in [12] also contributes a lot to the computational cost. As the modification factor is meant to reduce BSE, similar to the proposed IPD correction method, it also makes sense to study the results when no incident angle modification exists 


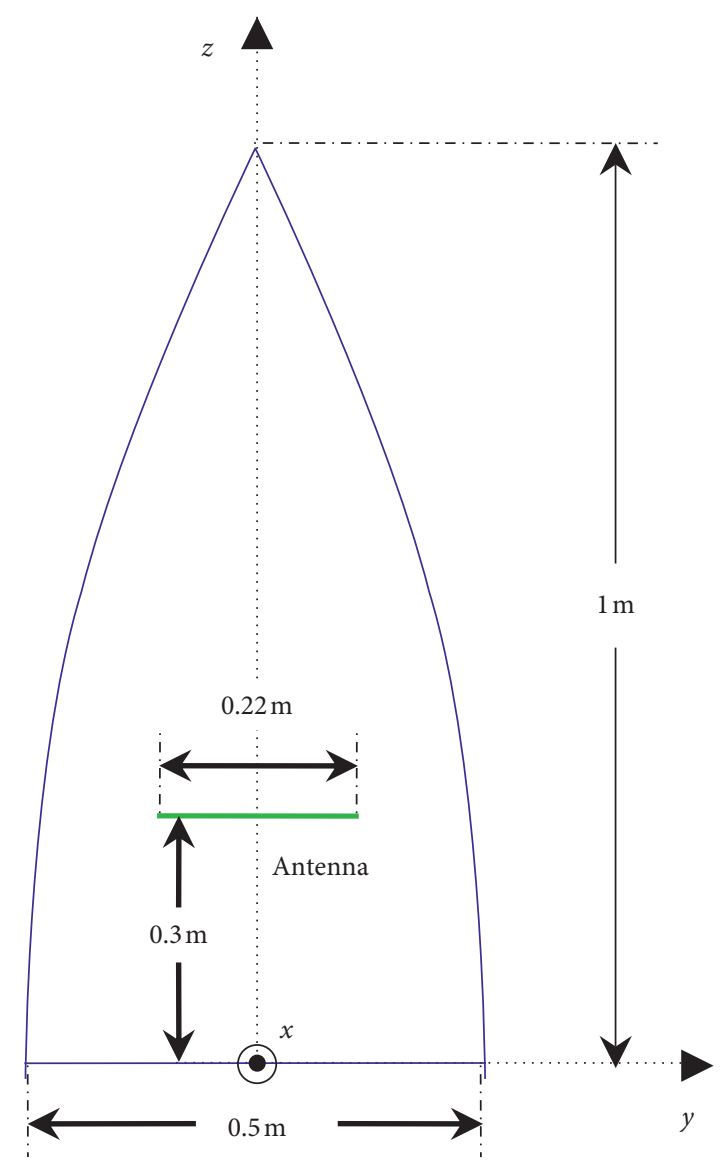

Figure 6: The tangent-ogival radome.

TABLE 1: The maximum BSE during IPD correction iteration.

\begin{tabular}{lcccc}
\hline Iteration & BSE $(\mathrm{mrad})$ & Scale factor & Side & TL $(\mathrm{dB})$ \\
\hline Initial & 1.388 & - & - & 0.53 \\
1 & 1.374 & 1.00 & Right & 0.53 \\
2 & 1.027 & 1.06 & Left & 0.53 \\
3 & 1.000 & 1.00 & Right & 0.53 \\
4 & 0.927 & 0.99 & Right & 0.53 \\
5 & 0.758 & 1.00 & Left & 0.53 \\
6 & 0.706 & 1.01 & Left & 0.53 \\
7 & 0.739 & 0.95 & Right & 0.54 \\
\hline
\end{tabular}

and only IPD correction works to improve BSE characteristics.

Table 4 lists the results when the IPD correction is imposed directly on the thickness profile obtained from the half-wave expression and no average incident angle modification exists. First, the previous factor range $[0.9,1.1]$ is adopted, and the optimal maximum BSE is $2.221 \mathrm{mrad}$, which is large. Considering that the modification factor 0.75 in [12] reduces the average incident angle and thus the thickness, the original thickness profile without modification factor is more different from the optimal thickness profile than the modified thickness profile. Therefore, it is considered that an extended scale factor range may help. Table 4 gives the results for scale factor range $[0.7,1.1]$. The reason why $[0.7,1.3]$ is not used is that from our many trials (not

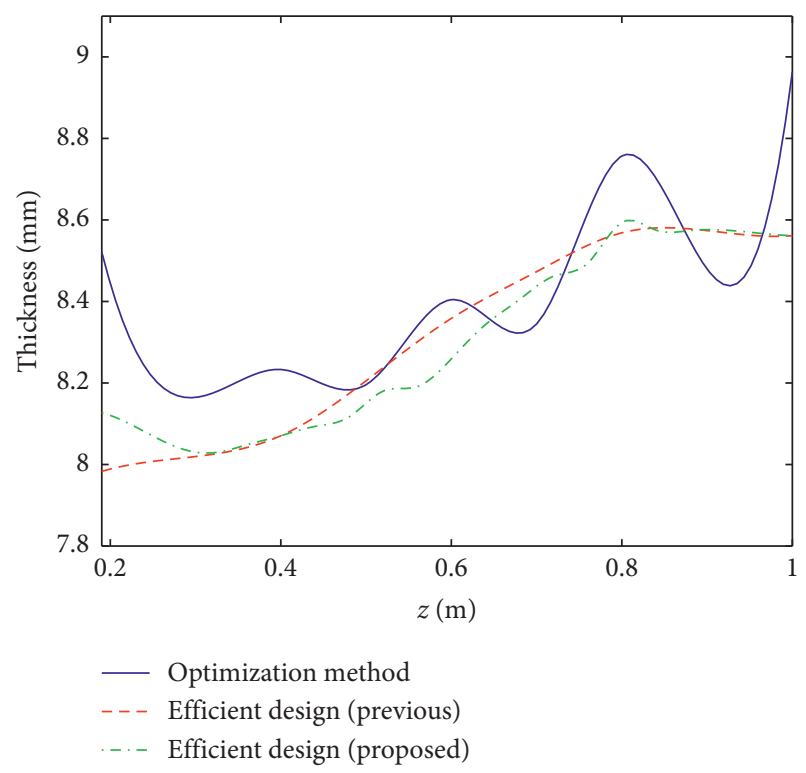

FIGURE 7: Thickness profile of VTRs obtained by different methods.

only the no-modification-factor case), the scale factor never exceeds 1.1. The optimal maximum BSE under this condition is $0.972 \mathrm{mrad}$, much better than the $[0.9,1.1]$ case but still inferior to the $0.706 \mathrm{mrad}$ in Section 3.1. Besides, the computational cost for this case is 651 EM performance 


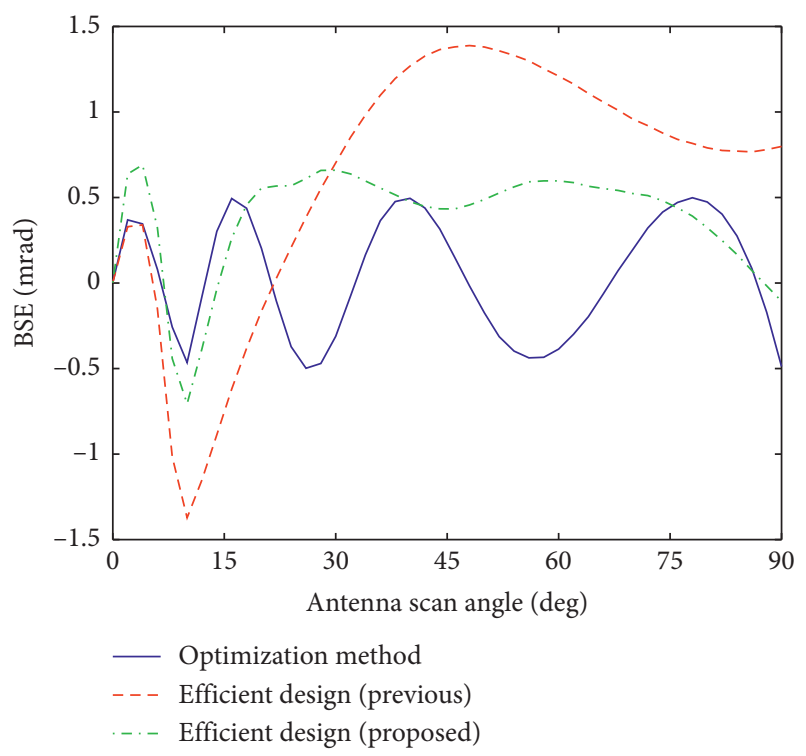

(a)

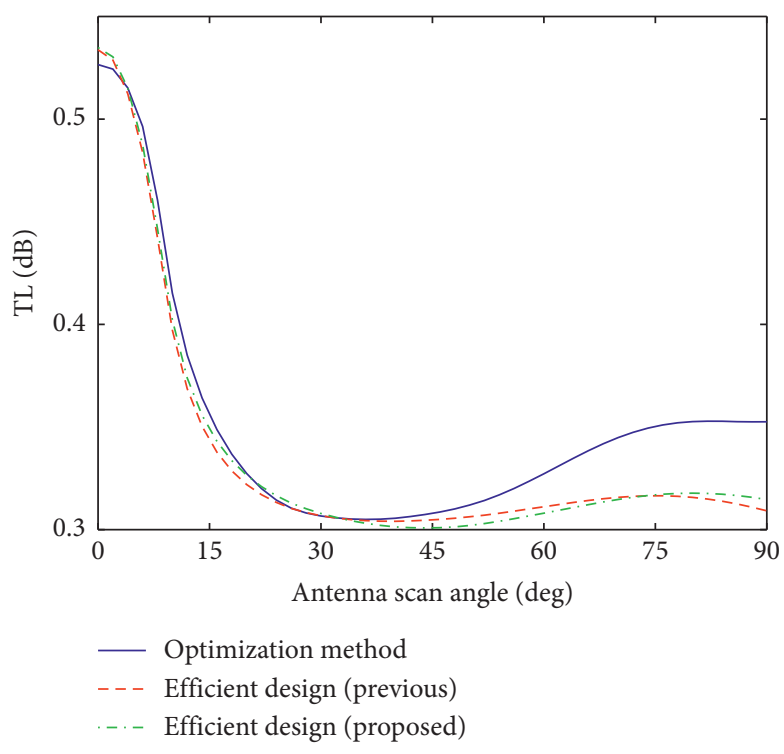

(b)

FiguRE 8: EM performance of VTRs obtained by different methods. (a) BSE. (b) TL.

TABLE 2: Results of different methods.

\begin{tabular}{|c|c|c|c|}
\hline & $\mathrm{BSE}_{\max }(\mathrm{mrad})$ & $\mathrm{TL}_{\max }(\mathrm{dB})$ & Number of EM computations \\
\hline Optimization method & 0.499 & 0.53 & 10000 \\
\hline Efficient design (previous) & 1.389 & 0.53 & 61 \\
\hline Efficient design (proposed) & 0.706 & 0.53 & 237 \\
\hline
\end{tabular}

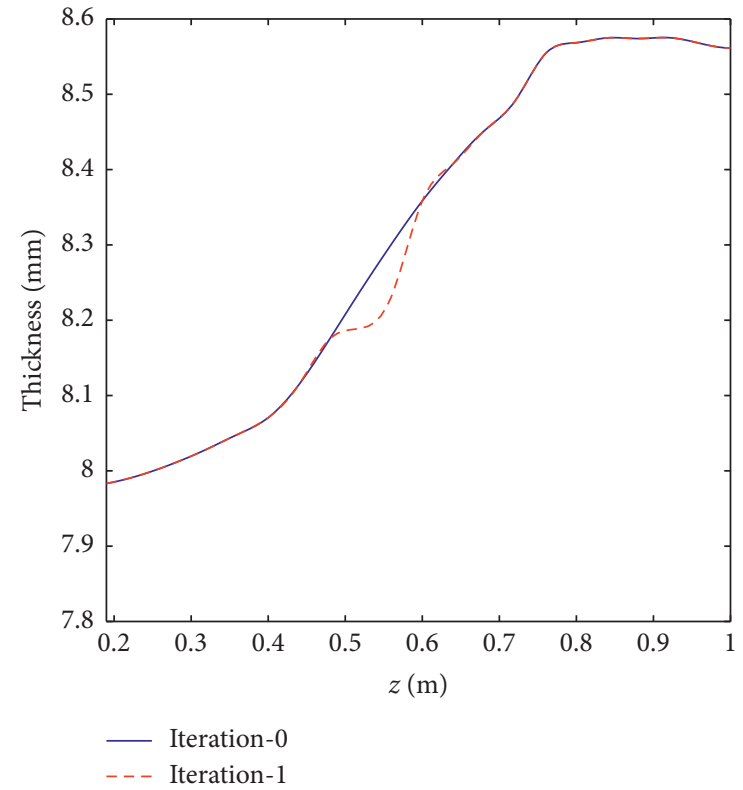

(a)

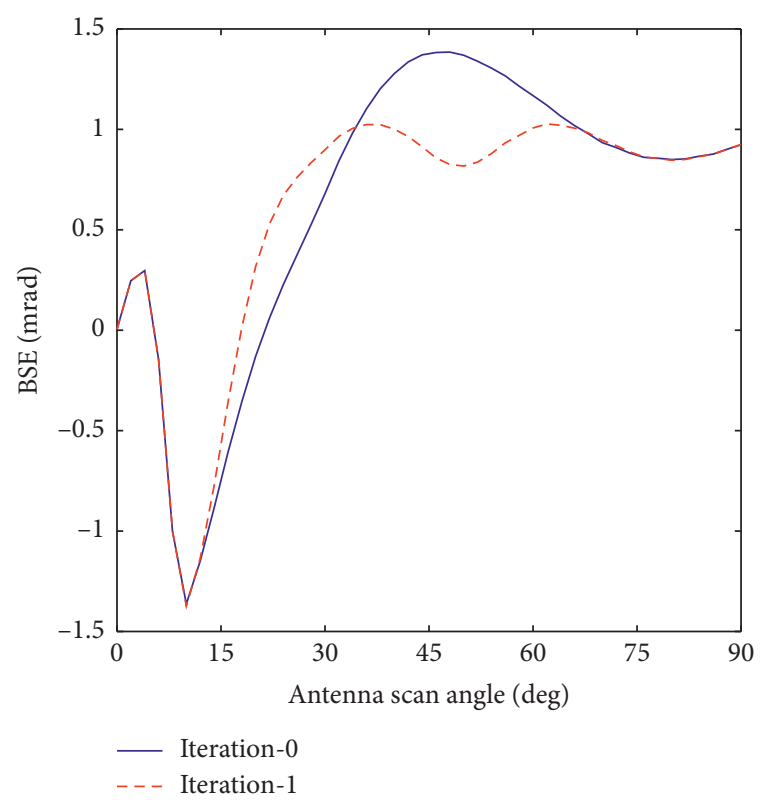

(b)

FIgURE 9: Continued. 

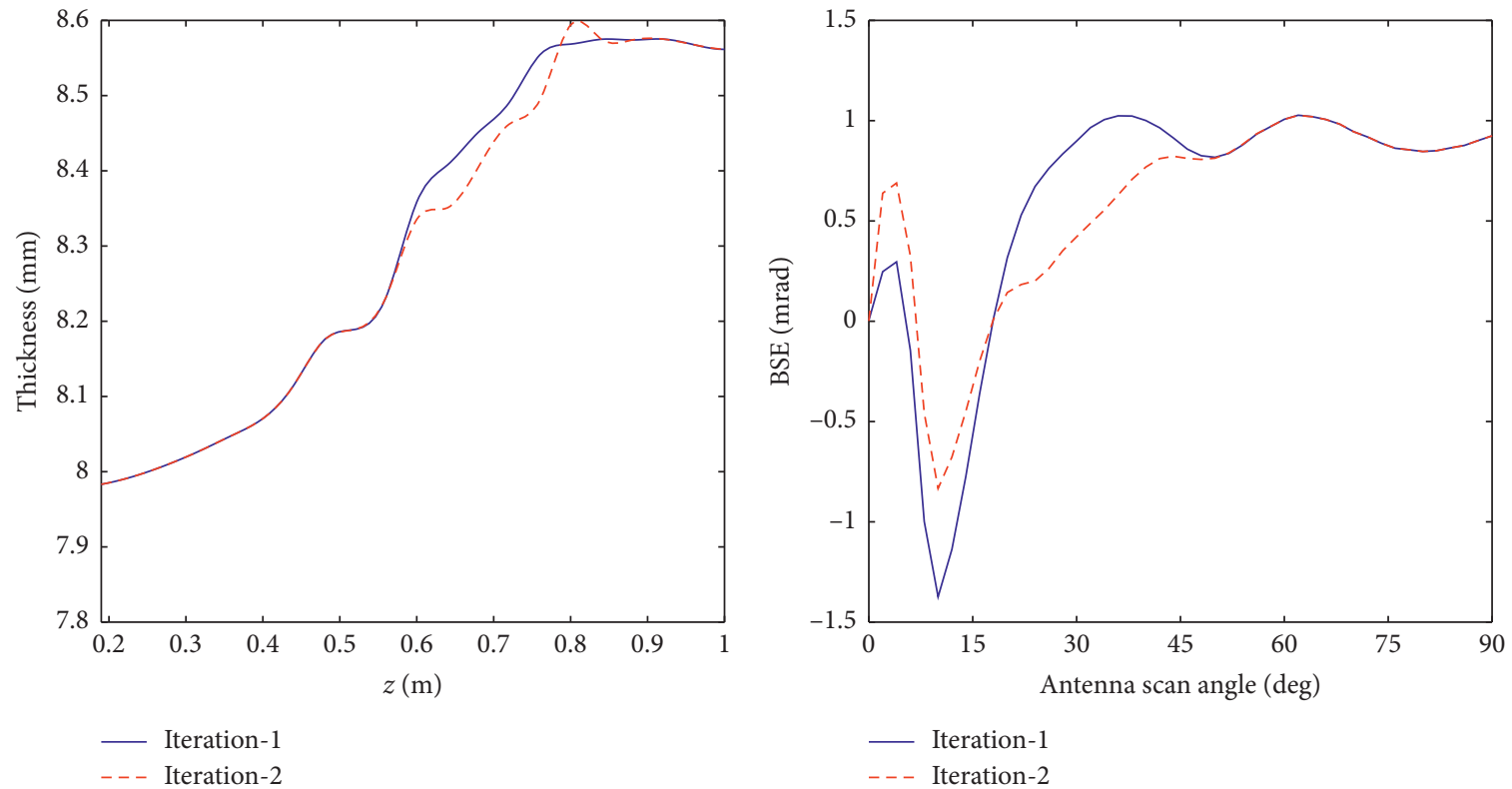

(c)

(d)
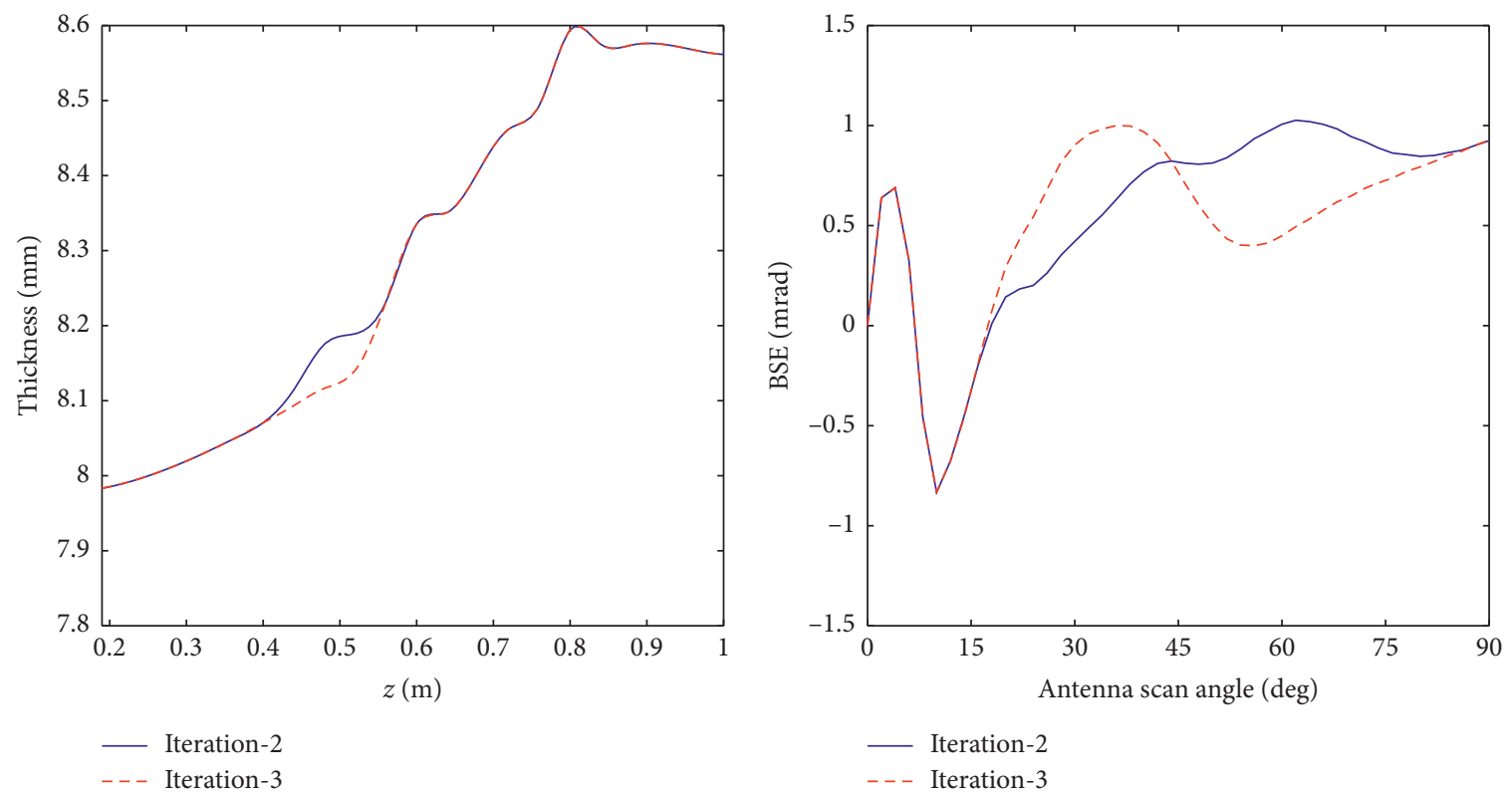

(e)

(f)

FIgUre 9: Continued. 


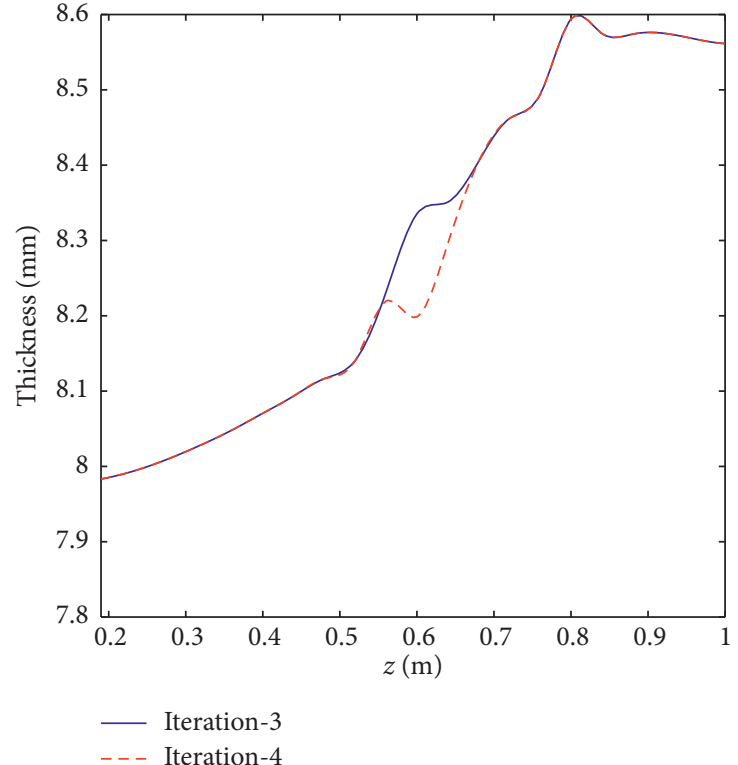

(g)

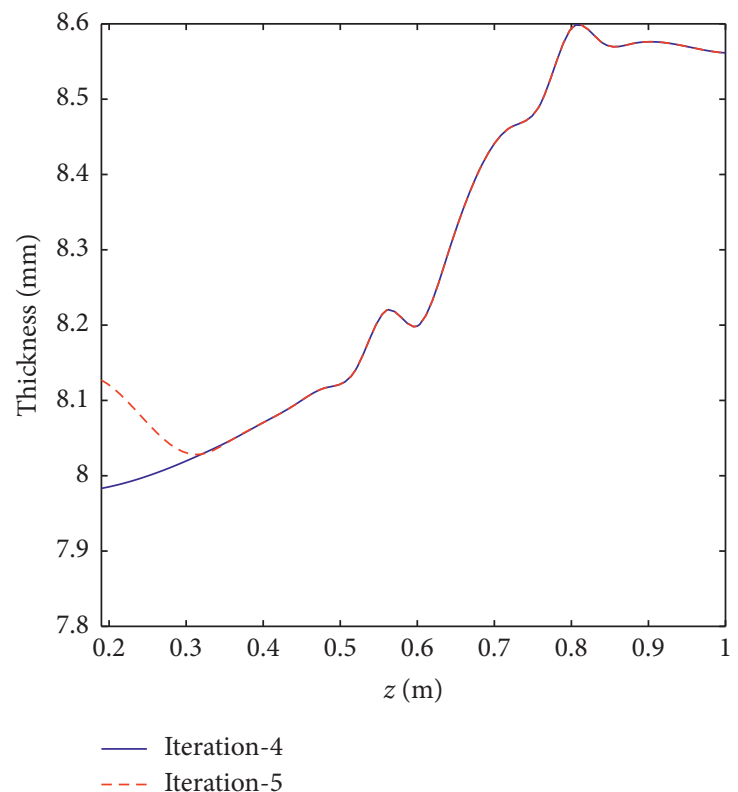

(i)

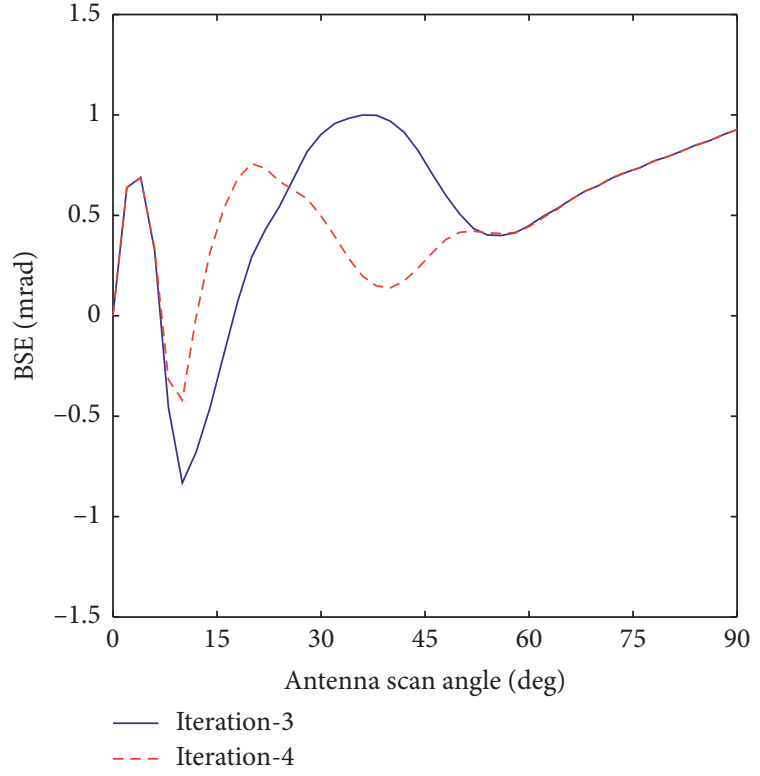

(h)

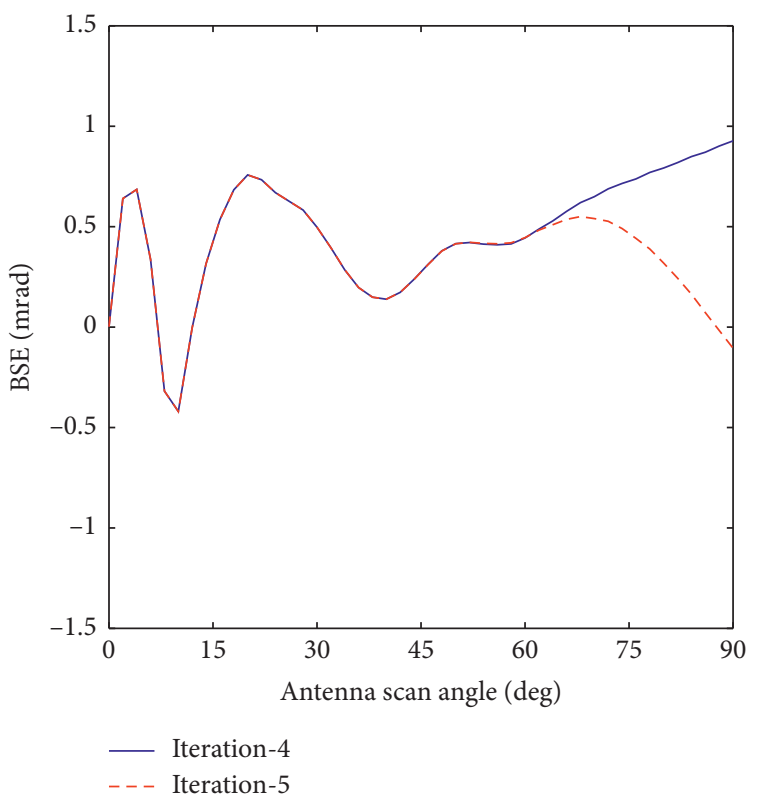

(j)

Figure 9: Continued. 


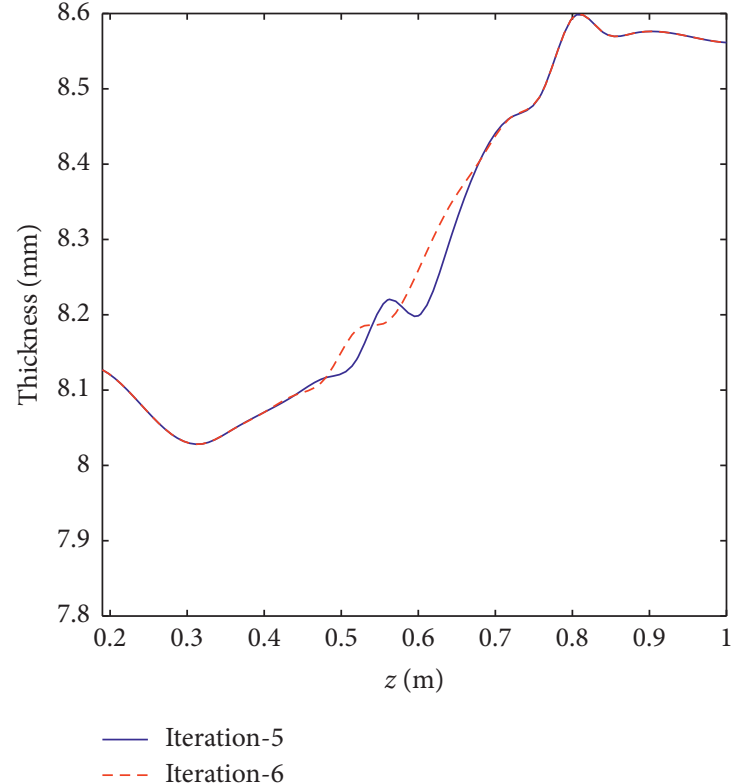

$(\mathrm{k})$

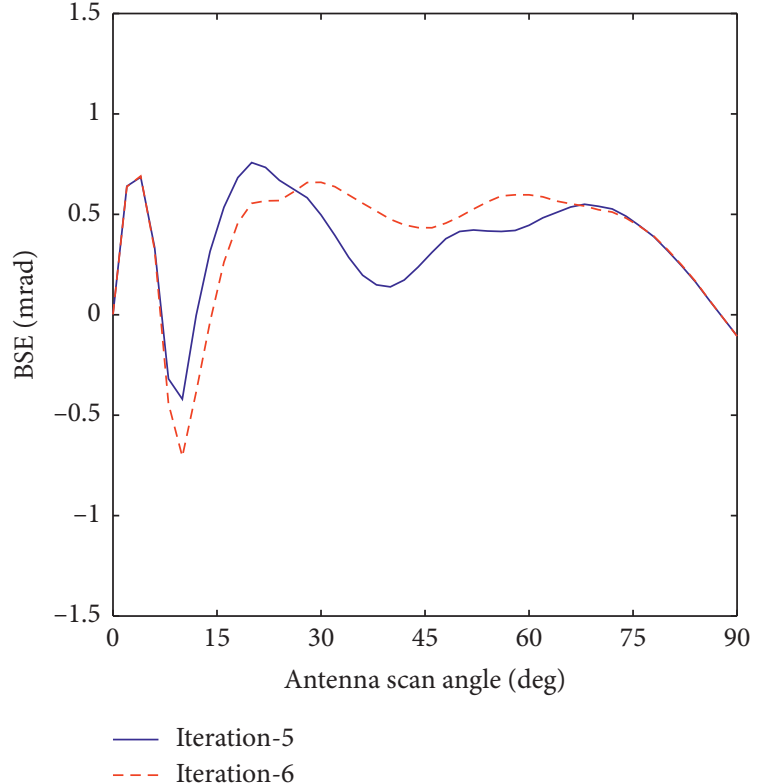

(l)

FIGURE 9: The thickness profile and BSE curve during the IPD correction process. (a, b) Iterations 0 and 1. (c, d) Iterations 1 and 2. (e, f) Iterations 2 and 3. (g, h) Iterations 3 and 4. (i, j) Iterations 4 and 5. (k, l) Iterations 5 and 6.

TABLE 3: The maximum BSE during IPD correction iteration for LSC/RSC.

\begin{tabular}{lccc}
\hline Iteration & \multicolumn{3}{c}{ BSE (mrad) } \\
\hline Initial & LSC & RSC \\
1 & & 1.388 & \\
2 & 1.397 & & 1.374 \\
3 & 1.373 & & 1.027 \\
4 & 1.363 & 1.083 \\
5 & 1.367 & 1.240 \\
6 & 1.401 & 1.199 \\
7 & 1.471 & 0.974 \\
8 & 1.399 & & 0.918 \\
\hline
\end{tabular}

TABLE 4: The maximum BSE during IPD correction iteration without incident angle modification factor.

\begin{tabular}{lcc}
\hline \multirow{2}{*}{ Iteration } & \multicolumn{3}{c}{ BSE (mrad) } \\
& Scale factor [0.9, 1.1] & Scale factor [0.7, 1.1] \\
\hline Initial & \multicolumn{2}{c}{2.919} \\
1 & 2.749 & 2.749 \\
2 & 2.574 & 2.247 \\
3 & 2.525 & 2.221 \\
4 & 2.476 & 2.039 \\
5 & 2.428 & 1.867 \\
6 & 2.379 & 1.796 \\
7 & 2.329 & 1.756 \\
8 & 2.281 & 1.466 \\
9 & 2.247 & 1.407 \\
10 & 2.232 & 1.065 \\
11 & 2.221 & 0.972 \\
12 & 2.354 & 1.102 \\
\hline
\end{tabular}

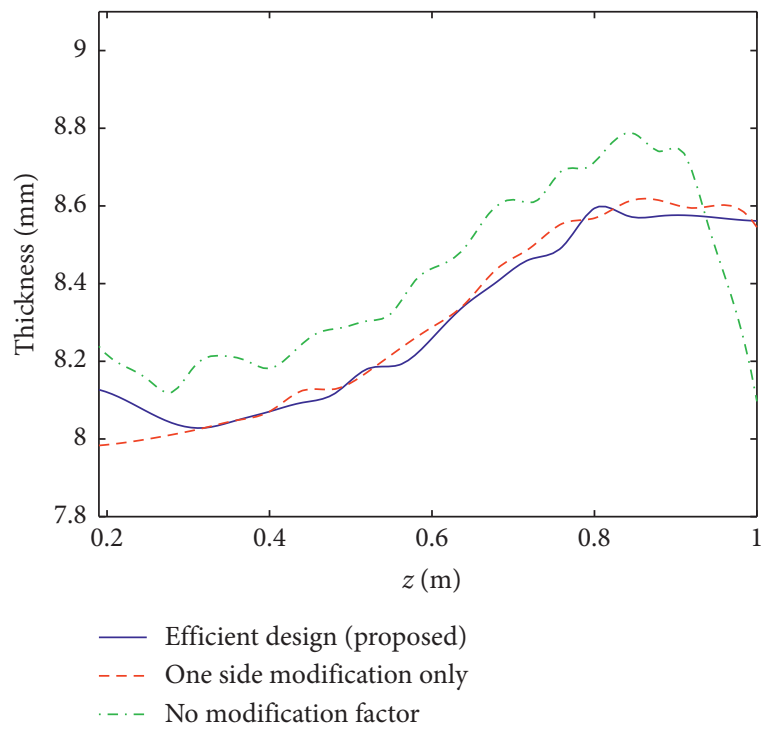

FIGURE 10: Thickness profile of VTRs obtained by the proposed method, one-side-correction-only method, and no-modificationfactor method.

calculations, much larger than the 237 times in the previous Section 3.1.

Figure 10 plots the thickness profile comparison of the proposed method, one-side-only method, and no-modification-factor method, and Figure 11 shows the corresponding EM performance comparison. Table 5 lists the EM performance and the computational cost.

From these results, it can be concluded that the proposed method has the overall best performance. Besides, some 


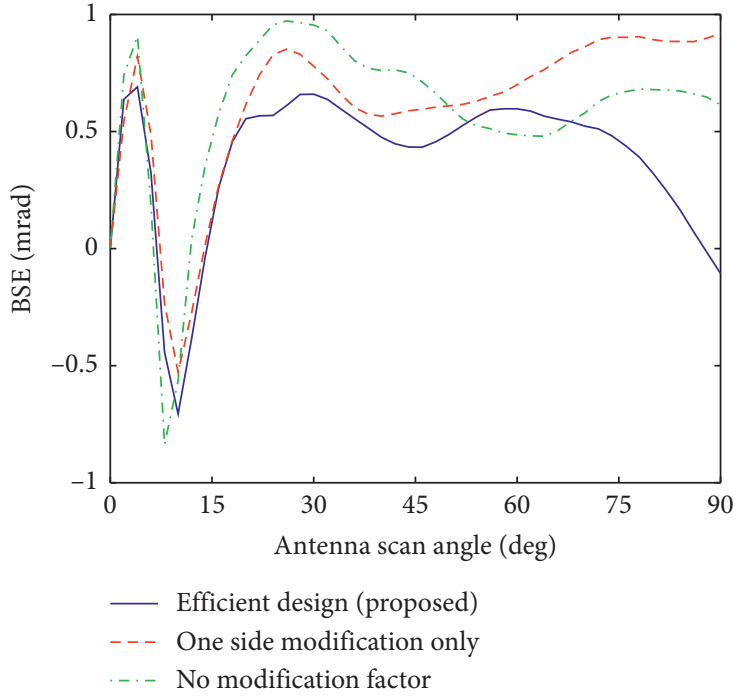

(a)

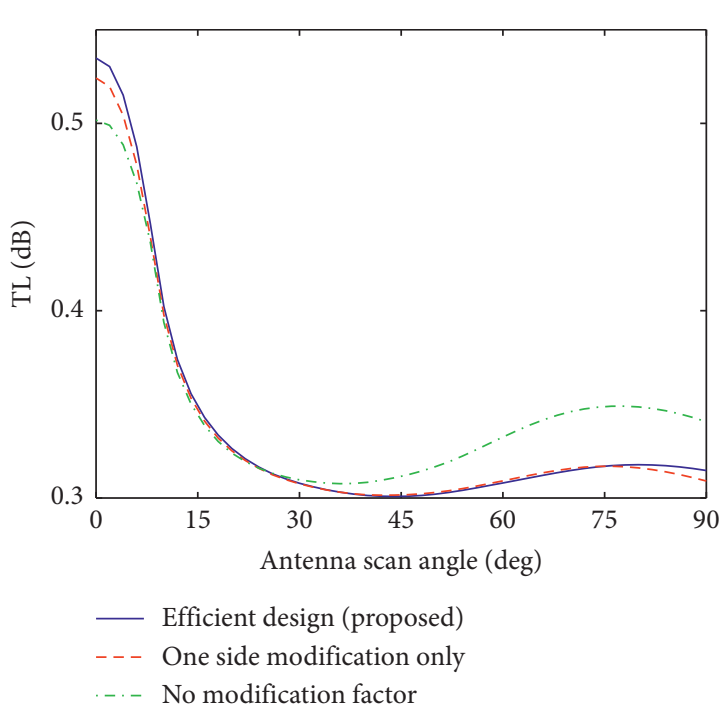

(b)

FIGURE 11: EM performance of VTRs obtained by the proposed method, one-side-correction-only method, and no-modification-factor method. (a) BSE. (b) TL.

TABLE 5: Effect of side selection and modification factor.

\begin{tabular}{lccc}
\hline & $\mathrm{BSE}_{\max }(\mathrm{mrad})$ & $\mathrm{TL}_{\max }(\mathrm{dB})$ & Number of EM computations \\
\hline Efficient design-proposed & 0.706 & 0.53 & 237 \\
One-side-correction only & 0.918 & 0.52 & 149 \\
No modification factor & 0.972 & 0.50 & 651 \\
\hline
\end{tabular}

hints can also be recognized. In the proposed method, the adoption of the modification factor in fact makes the thickness profile obtained by half-wave expression approach the optimal thickness profile with better BSE characteristics, which is like a coarse-adjustment step, and the LSC-RSC method implements the fine-adjustment step.

\section{Conclusion}

In this work, IPD correction is introduced in the efficient thickness profile design of streamlined airborne radomes. Based on the half-wave wall design from average incident angle with a modification factor, the thickness profile is further adjusted for IPD symmetry at particular antenna scan angles. Both left-side correction (LSC) and right-side correction (RSC) are considered in IPD correction. Several conclusions can be drawn:

(1) The proposed method can well improve the BSE characteristics of the half-wave wall design with slight increase of computational cost and deterioration of thickness profile smoothness. The scale factor range $[0.9,1.1]$ with a step of 0.01 is suggested.

(2) The modification factor and LSC-RSC method are both important in realizing the good performance, and the former essentially works as a coarse-adjustment step while the latter as a fine-adjustment step.
(3) Using the proposed method, the VTR can be efficiently designed with EM performance close to the traditional optimization design and thickness profile nearly as smooth as the efficient average incident angle method. A better balance between EM performance and thickness profile smoothness of airborne radomes is achieved.

\section{Data Availability}

All data generated or analyzed during this study are included in this article.

\section{Conflicts of Interest}

The authors declare that they have no conflicts of interest.

\section{Acknowledgments}

This work was supported by the National Natural Science Foundation of China (Grant nos. 51605362, 51875431, and 51775405), Natural Science Basic Research Plan in Shaanxi Province of China (Grant no. 2018JQ5063), the Fundamental Research Funds for the Central Universities, and the 111 Project (B14042).

\section{References}

[1] D. J. Kozakoff, Analysis of Radome-Enclosed Antennas, Artech House, Boston, MA, USA, 2nd edition, 2010. 
[2] C. S. Wang, B. Y. Duan, and Y. Y. Qiu, "On distorted surface analysis and multidisciplinary structural optimization of large reflector antennas," Structural and Multidisciplinary Optimization, vol. 33, no. 6, pp. 519-528, 2007.

[3] B. Y. Duan and C. S. Wang, "Reflector antenna distortion analysis using MEFCM," IEEE Transactions on Antennas and Propagation, vol. 57, no. 10, pp. 3409-3413, 2009.

[4] C. S. Wang, B. Y. Duan, F. S. Zhang, and M. B. Zhu, "Coupled structural-electromagnetic-thermal modelling and analysis of active phased array antennas," IET Microwaves, Antennas \& Propagation, vol. 4, no. 2, pp. 247-257, 2010.

[5] R. U. Nair and R. M. Jha, "Electromagnetic performance analysis of a novel monolithic radome for airborne applications," IEEE Transactions on Antennas and Propagation, vol. 57, no. 11, pp. 3664-3668, 2009.

[6] F. Hsu, S. H. Chao, K. K. Chan, and P. R. Chang, "Optimal boresight error design of radomes of revolving symmetry," Electronics Letters, vol. 30, no. 19, pp. 1561-1562, 1994.

[7] F. Hsu, P.-R. Chang, and K.-K. Chan, "Optimization of twodimensional radome boresight error performance using simulated annealing technique," IEEE Transactions on Antennas and Propagation, vol. 41, no. 9, pp. 1195-1203, 1993.

[8] W. Xu, B. Y. Duan, P. Li, and Y. Qiu, "Study on the electromagnetic performance of inhomogeneous radomes for airborne applications-part I: characteristics of phase distortion and boresight error," IEEE Transactions on Antennas and Propagation, vol. 65, no. 6, pp. 3162-3174, 2017.

[9] W. Xu, B. Y. Duan, P. Li, and Y. Qiu, "Study on the electromagnetic performance of inhomogeneous radomes for airborne applications-part II: the overall comparison with variable thickness radomes," IEEE Transactions on Antennas and Propagation, vol. 65, no. 6, pp. 3175-3183, 2017.

[10] H. Meng and W. Dou, "Multi-objective optimization of radome performance with the structure of local uniform thickness," IEICE Electronics Express, vol. 5, no. 20, pp. $882-887,2008$

[11] W. Xu, B. Y. Duan, P. Li, N. Hu, and Y. Qiu, "Multiobjective particle swarm optimization of boresight error and transmission loss for airborne radomes," IEEE Transactions on Antennas and Propagation, vol. 62, no. 11, pp. 5880-5885, 2014.

[12] W. Xu, B. Y. Duan, P. Li, and Y. Qiu, "A new efficient thickness profile design method for streamlined airborne radomes," IEEE Transactions on Antennas and Propagation, vol. 65, no. 11, pp. 6190-6195, 2017.

[13] P. S. M. Yazeen, C. V. Vinisha, S. Vandana, M. Suprava, and R. U. Nair, "Electromagnetic performance analysis of graded dielectric inhomogeneous streamlined airborne radome," IEEE Transactions on Antennas and Propagation, vol. 65, no. 5, pp. 2718-2723, 2017. 


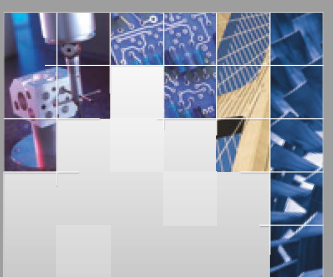

\section{Enfincering}
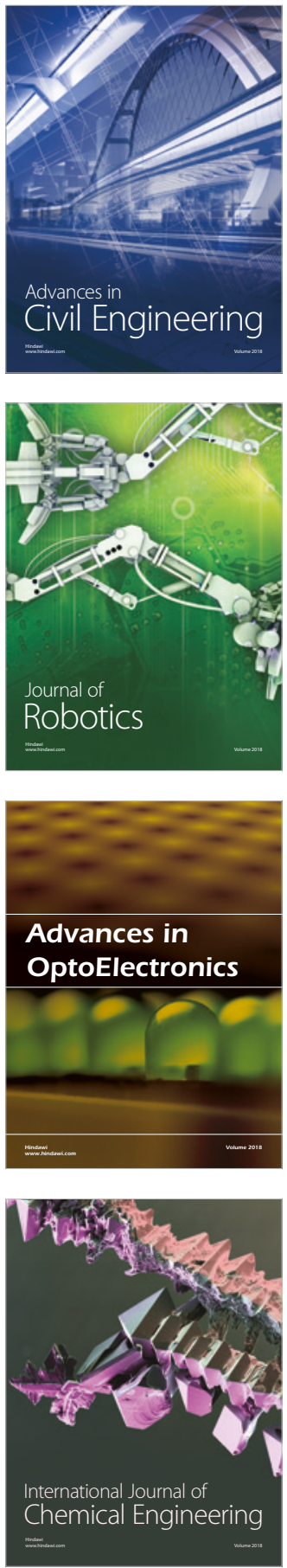

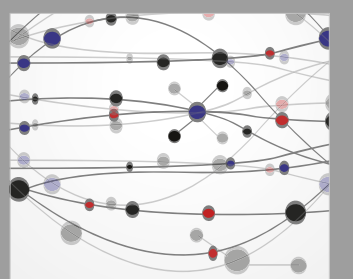

\section{Rotating \\ Machinery}

The Scientific World Journal

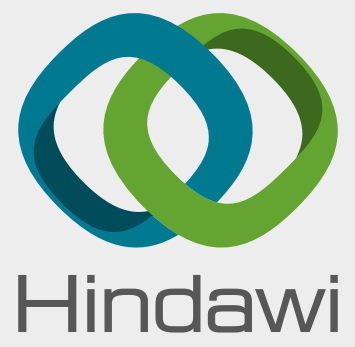

Submit your manuscripts at

www.hindawi.com
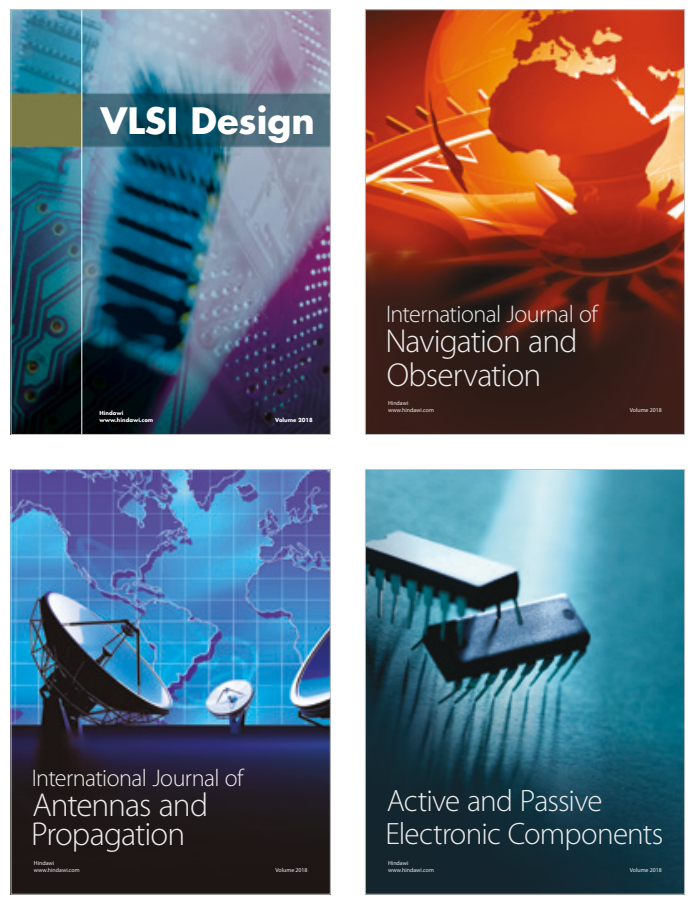
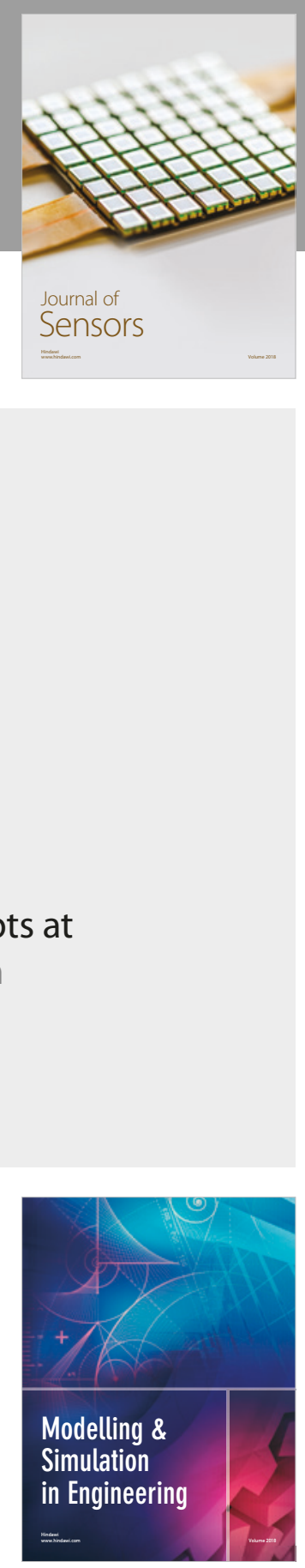

\section{Advances \\ Multimedia}
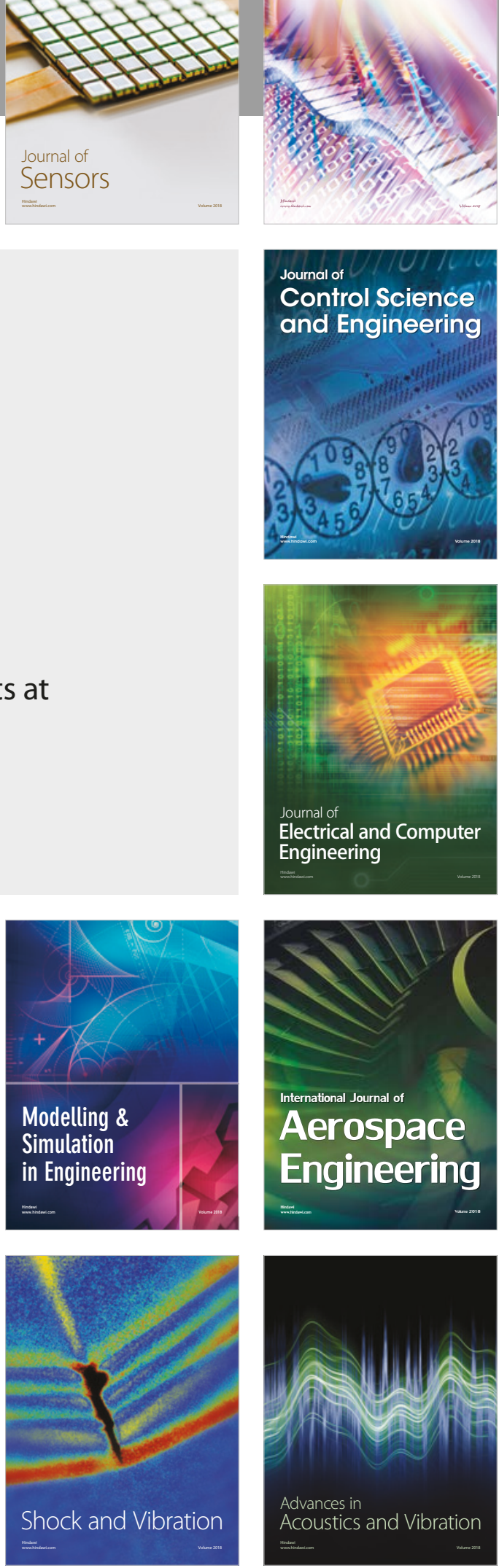